\title{
WAVE PATTERN DYNAMICS IN FLUCTUATING MEDIA*
}

\author{
F. SAGUÉS ${ }^{\dagger}$ and S. ALONSO $\ddagger$ \\ Departament de Química Física, Universitat de Barcelona \\ Diagonal 647, 08028, Barcelona, Spain \\ ${ }^{\dagger}$ f.sagues@qf.ub.es \\ $\ddagger_{s . a l o n s o @ q f . u b . e s}$ \\ J. M. SANCHO \\ Departament d'Estructura $i$ Constituents de la Matèria \\ Facultat de Física, Universitat de Barcelona, Diagonal 647 \\ 08028, Barcelona, Spain \\ jmsancho@ecm.ub.es
}

\begin{abstract}
Analytical and numerical results on the ordering role of external random fluctuations in excitable systems are presented. Our study focuses on a simple model for excitable systems. Regular waves are created and sustained out of noise when the system is forced with random perturbations. Explicit results for the generation and dynamics of rings and targets are presented.
\end{abstract}

Keywords: Stochastic; excitable media; nonlinear dynamics; target patterns.

\section{Introduction}

We do know now that stochastic environments or external fluctuations can generate counter-intuitive effects such as ordered macroscopic phases, patterns, regular oscillations, etc. ${ }^{1,2}$ Physical mechanisms of such ordering role of the stochasticity have been elucidated and explained in simple modelizations. The most commonly accepted origin of this unexpected phenomenon is the cooperative interaction between fluctuations, nonlinearities and spatial coupling.

We will focus here in the so-called excitability scenario in which wave propagation appears naturally. There is a rich variety of physical, ${ }^{3,4}$ chemical, ${ }^{5,6}$ or biological $^{7}$ among other systems which present an excitable regime exhibiting wave propagation phenomenology. These systems show a large diversity of spatiotemporal patterns, ranging from planar waves to much more complicated structures such as targets or scroll rings. Similar problems are discussed in the contribution by L. Schimansky-Geier in this paper.

A theoretical modelization based on Stochastic Partial Differential Equations (SPDE) is the most common tool for the description and study of wave dynamics

*This contribution has been prepared to honor the 50th anniversary of Prof. J. Luczka. 
in fluctuating media. ${ }^{8}$ The question of integration of SPDE is also presented in the contribution by R. Mannella. Numerical simulations of these type of equations do show clearly the ordering effects induced by noise. Analytical, although nonexact, calculations also prove the possible existence of transitions from disorder to order as the intensity of the fluctuations is increased. This surprising scenario has recently received an important experimental support by a series of experimental evidences of these counter-intuitive ordering effects out of noise under well-controlled laboratory setups. $^{9-12}$

Our objective in this paper is to present the theoretical approach to SPDE's, with special emphasis in its predictive strength. Numerical simulations are used as a test of the reliability and accuracy of our treatment, because a more rigorous mathematical approach to SPDE's is not yet available.

This paper is organized as follows. In Sec. 2 we present the main aspects of our analytical and numerical methodology. Section 3 contains the explicit results for a very simple modelization of an excitable system. Finally we end up with some conclusions and perspectives.

\section{Modelization and Methodology}

\subsection{Stochastic Partial Differential Equations (SPDE)}

The common framework to model pattern formation phenomenology is by the use of partial differential equations (PDE) for the local densities of the material components. The most simple situation which presents waves has two variables called activator, $u(\mathbf{x}, t)$, and inhibitor, $v(\mathbf{x}, t)$, with different time scales, $u(\mathbf{x}, t)$, is the fast variable. These equations have the general form,

$$
\begin{aligned}
& \partial_{t} u=D \nabla^{2} u+f_{u}(u, v, b), \\
& \partial_{t} v=f_{v}(u, v)
\end{aligned}
$$

where $f_{i}$ are the reactive terms and, quite often, it is assumed that the inhibitor variable diffuses so slowly that one can discard its contribution. The conditions $f_{i}=0$ determine the null-clines whose intersection points give the homogeneous stationary states of the system. The relative stability or nature of these points can be obtained by a simple linear analysis. $b$ is an external parameter which controls the excitability properties of the medium. In the experiments on the photosensitive Belousov-Zhabotinsky reaction, the parameter $b$ will represent the intensity of the incident light. ${ }^{9-12}$ This parameter can be handled easily by using opto-electronic devices and, as a consequence, controlled fluctuations can be introduced through it as, $b \rightarrow b+\eta(\mathbf{x}, t)$. Then one can arrive to the following generic system of stochastic partial differential equations (SPDE) which will be the framework of all our studies,

$$
\begin{aligned}
& \partial_{t} u=D \nabla^{2} u+f_{u}(u, v, b)+g_{u}(u) \eta(\mathbf{x}, t), \\
& \partial_{t} v=f_{v}(u, v)
\end{aligned}
$$


where $\eta(\mathbf{x}, t)$ is a Gaussian noise with zero mean and a correlation,

$$
\left\langle\eta\left(\mathbf{x}^{\prime}, t^{\prime}\right) \eta(\mathbf{x}, t)\right\rangle=2 \sigma^{2} \delta\left(t-t^{\prime}\right) \delta\left(\mathbf{x}-\mathbf{x}^{\prime}\right) .
$$

Now depending on the modelization involved in a particular system, different reactive functions $f_{i}$ and $g_{i}$, are proposed.

The set of Eqs. (2)-(3) with appropriate boundary and initial conditions define the system we want to study. Thus, we deal with nonlinear SPDE's whose analytical solution is impossible. Nevertheless analytical information is highly valuable and, as a consequence, some efforts should be invested in this direction. Numerical simulations of these equations have to complete this study.

\subsection{Effective dynamics}

Here we will outline the most suitable theoretical approach to understand how ordering effects out of fluctuations could appear. The idea is very simple. The initial conjecture is that fluctuations not only have a dispersive or disordering effects but also the systematic ones. If it is so, one can try to guess where is the source of them. A preliminary analysis of Eq. (2) shows that the noisy term $g(u)_{u} \eta(\mathbf{x}, t)$ has a nonzero mean value given by:

$$
\left\langle g_{u}(u) \eta(\mathbf{x}, t)\right\rangle \equiv\langle\Phi(u)\rangle \neq 0,
$$

in spite of the zero average of the noise. We are using the Stratonovich calculus to deal with the multiplicative noise. This is the usual interpretation when we face with realistic noise. The above contribution will be the source of the systematic and quite often ordering effects of the noise. ${ }^{1}$ By adding and subtracting $\Phi(u)$ to the equation of motion for $u$ in Eq. (2), we arrive at:

$$
\begin{aligned}
& \partial_{t} u=D \nabla^{2} u+f_{u}(u, v, b)+\Phi(u)+R(u, t), \\
& \partial_{t} v=f_{v}(u, v) .
\end{aligned}
$$

$R(u, t)=g(u) \eta(\mathbf{x}, t)-\Phi(u)$, is a more complicated random term which has now a zero mean value and whose fluctuating and nonsystematic effects can be safely discarded for not very large noise intensities. Then one arrives to the effective deterministic model,

$$
\begin{aligned}
& \partial_{t} u=D \nabla^{2} u+f_{u}(u, v, b)+\Phi(u), \\
& \partial_{t} v=f_{v}(u, v)
\end{aligned}
$$

which can be studied using the standard techniques for PDE's. In the explicit example we will study in the next section, the new reactive term $\Phi(u)$ can be absorbed in the old one $f_{u}(u, v, b)$ through a renormalization of the model parameters in such a way that its systematic influence can be evaluated using already developed techniques for PDE's.

Our analytical approach relays on the evaluation of $\Phi(u)$. This is a simple task for a white noise ${ }^{1}$ but it is much involved for spatio-temporal structured noise. ${ }^{13}$ 


\subsection{Numerical simulations}

In order to see how is the phenomenology of systems described by SPDE's such as Eqs. (2) and (3) and also to test the accuracy of the theoretical predictions we have to proceed with the numerical simulation of this type of equations. We will follow the Heun method (a second order Runge-Kutta algorithm) extended for SPDE's. ${ }^{1}$ The set of Eqs. (2) and (3) are discretized in a d-dimensional lattice with $N$ squared cells, each one of the linear size $\Delta x$. The fields $u(\mathbf{x}, t)$ and $v(\mathbf{x}, t)$ transform into $N$ time-dependent variables $u_{i}(t)$ and $v_{i}(t)$ where $i$ is the position index. In the discrete lattice we have $N$ couples of ordinary stochastic differential equations. The Laplacian operator transform according to the standard prescription,

$$
D \nabla^{2} u \rightarrow \mathcal{D}_{i}(u)=\frac{D}{\Delta x^{2}}\left(\sum_{j} u_{j}(i)-2 d u_{i}\right),
$$

where $u_{j}(i)$ are the $2 d$ next neighbors of $u_{i}$. The noise is also discretized accordingly, $\eta(\mathbf{x}, t) \rightarrow \eta_{i}(t)$, with a correlation,

$$
\left\langle\eta_{i}(t) \eta_{j}(t)\right\rangle=2 \sigma^{2} \delta\left(t-t^{\prime}\right) \frac{\delta_{i j}}{\Delta x^{d}} .
$$

Now the Heun algorithm reads explicitly,

$$
\begin{aligned}
u_{i}(t+\Delta t)= & u_{i}(t)+\frac{\Delta t}{2}\left(f_{u}(u, v)+f_{u}(\tilde{u}, \tilde{v})+\mathcal{D}_{i}(u)+\mathcal{D}_{i}(\tilde{u})\right) \\
& +\frac{1}{2}\left(g(u)_{u}+g(\tilde{u})_{u}\right) X_{i} \\
v_{i}(t+\Delta t)= & v_{i}(t)+\frac{\Delta t}{2}\left(f_{v}(u, v)+f_{v}(\tilde{u}, \tilde{v})\right),
\end{aligned}
$$

where the predictor values $\tilde{u}$ and $\tilde{v}$ are obtained from the Euler algorithm,

$$
\begin{aligned}
& \tilde{u}_{i}=u_{i}(t)+\Delta t\left(f_{u}(u, v)+\mathcal{D}_{i}(u)\right)+g(u)_{u} X_{i}, \\
& \tilde{v}_{i}=v_{i}(t)+\Delta t f_{v}(u, v) .
\end{aligned}
$$

$X_{i}$ is random process given by:

$$
X_{i}=\sqrt{\frac{2 \sigma^{2} \Delta t}{\Delta x^{d}}} \gamma_{i}
$$

where $\gamma_{i}$ are Gaussianly distributed random numbers with zero mean and variance equal to 1 . This noise prescription generates a Gaussian white noise in a lattice.

The simulations have been done either in a one- or two-dimensional lattices with appropriate $\Delta x$ and $\Delta t$ which will be indicated in the figure captions corresponding to the numerical results.

Choosing appropriate initial conditions for each case, respectively a circular spot, ring, spiral or a planar wave, one can observe the dynamics of a particular structure under the presence of the external fluctuations. 


\section{The Barkley Model}

\subsection{Explicit predictions}

Our theoretical approach can be applied to any FitzHugh-Nagumo-like schemes to describe active systems. For the sake of simplicity and unless it is explicitly said, we will use Barkley modelization for a generic active media. ${ }^{14}$ This model has simple polynomial reactive terms,

$$
\begin{aligned}
f_{u}(u, v, b) & =\frac{1}{\varepsilon} u(1-u)\left(u-\frac{v+b}{a}\right), \\
f_{v}(u, v) & =u-v,
\end{aligned}
$$

where $\varepsilon$ controls the time scale of the activator and $a$ is another system parameter. Including the fluctuations in $b$ the multiplicative noise reactive term is:

$$
g_{u}(u)=\frac{1}{a \varepsilon} u(1-u) .
$$

Explicit calculations of the stochastic average defined in Eq. (4), using the Stratonovich interpretation, gives ${ }^{1,13}$

$$
\left\langle g_{u}(u) \eta(\mathbf{x}, t)\right\rangle=C(0)\left\langle g_{u}(u) g_{u}^{\prime}(u)\right\rangle,
$$

where $C(0)=\sigma^{2} / \Delta x^{2}$ is the effective noise intensity in the lattice. This gives, for our model,

$$
\Phi(u)=\frac{C(0)}{a^{2} \varepsilon^{2}} u(u-1)(2 u-1) .
$$

This systematic contribution is incorporated into the activator equation as a new reactive term. Finally the whole reactive term can be reorganized and it happens that the new effective reactive term is of the same form as the original deterministic one, but with the following new model parameters,

$$
a^{\prime}=a-\frac{2 C(0)}{a \varepsilon}, \quad b^{\prime}=b-\frac{C(0)}{a \varepsilon}, \quad \varepsilon^{\prime}=\frac{\varepsilon}{1-2 C(0) / a^{2} \varepsilon} .
$$

As parameter $b$ controls the excitability of the system one can expect important changes. In particular the decrease of $b$ as the noise intensity increases will drive the system to more excitable regimes. These aspects will appear clearly in our simulations.

\subsection{Numerical simulation results}

First we will study the transition between a nonexcitable regime and the excitable one. In a nonexcitable regime, any initial pattern or perturbation will decay to the uniform state $u=v=0$, and no propagation is observed. Under this conditions, we can see how increasing the intensity of the noise will arrive to an excitable regime in which the initial pattern is sustained and propagates. In Fig. 1 we find this behavior in numerical simulations. The first column in Fig. 1 has been included to 


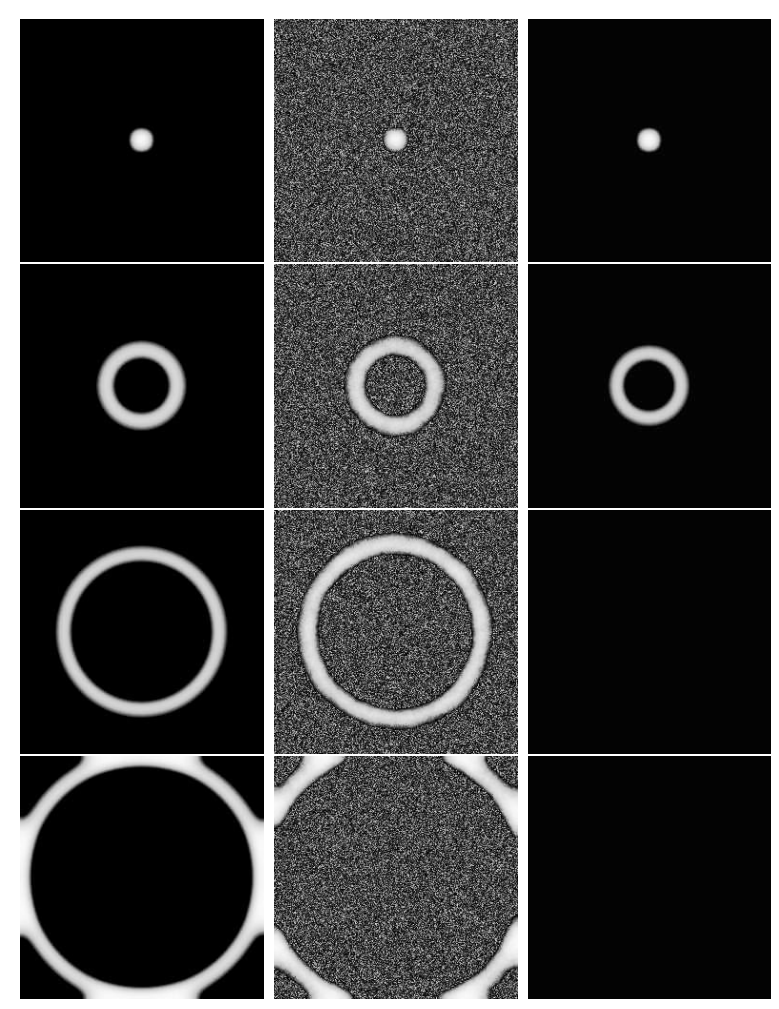

Fig. 1. Two-dimensional sub-excitable system with a circular-like initial condition. The first column corresponds to the effective model with $C(0)=0.001$. The second one corresponds to the same system with white noise $\left(\sigma^{2}=0.00001\right)$. The last column corresponds to the deterministic model in the sub-excitable regime. There are 3 t.u. between each snapshot $(512 \times 512$ pixels, $a=0.7, b=0.02, \epsilon=0.07, \Delta x=0.1$ s.u., $\Delta t=0.001$ t.u. $)$.

appreciate the effective model mimics qualitatively and quantitatively the behavior found in simulations.

The transition from an excitable to an oscillatory regime can be predicted from the study of the null-clines. In Fig. 2 plots of the null-clines and stationary points are presented for the deterministic and stochastic effective models.

One can see how the stability of one of the steady points change with the presence of fluctuations. In particular the deterministically stable steady state in an excitable regime transforms, due to the presence of noise in the unstable steady point of an oscillatory regime.

This theoretical prediction has been tested by numerical simulations and a representative evolution is presented in Fig. 3. Here we observe how a system in an excitable regime of expanding rings without noise, changes to an oscillatory regime with concentric rings (targets) when noise is present. As in the former case, the first column in Fig. 3 shows that the effective deterministic model explains indeed this result. 


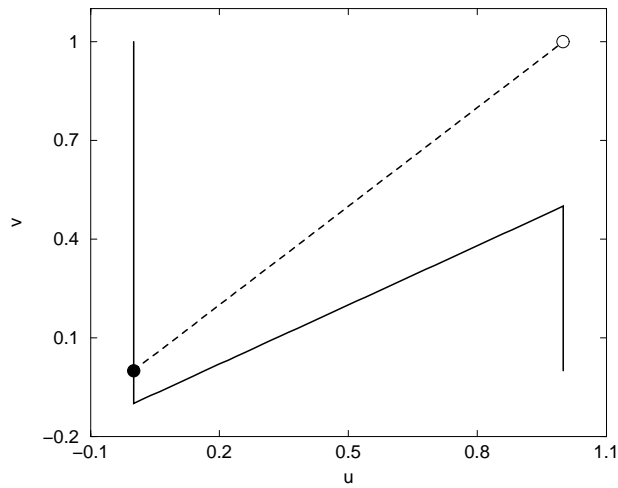

(a)

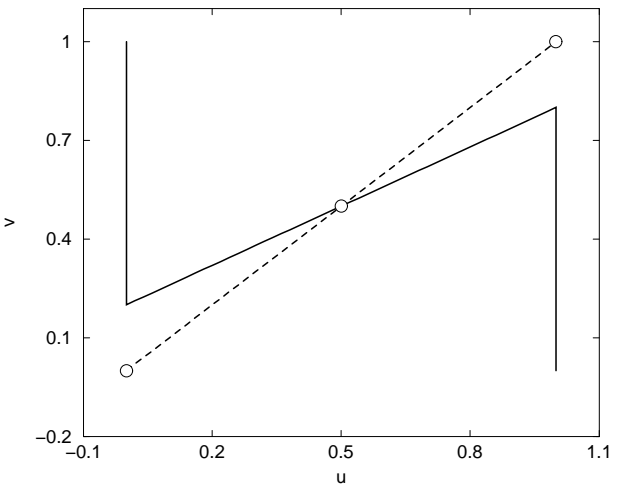

(b)

Fig. 2. (a) Null-clines of the deterministic model (12). (b) Null-clines of the effective deterministic model: Eq. (12) with parameters (16). Empty circles are the unstable steady points and full circles are the stable ones.

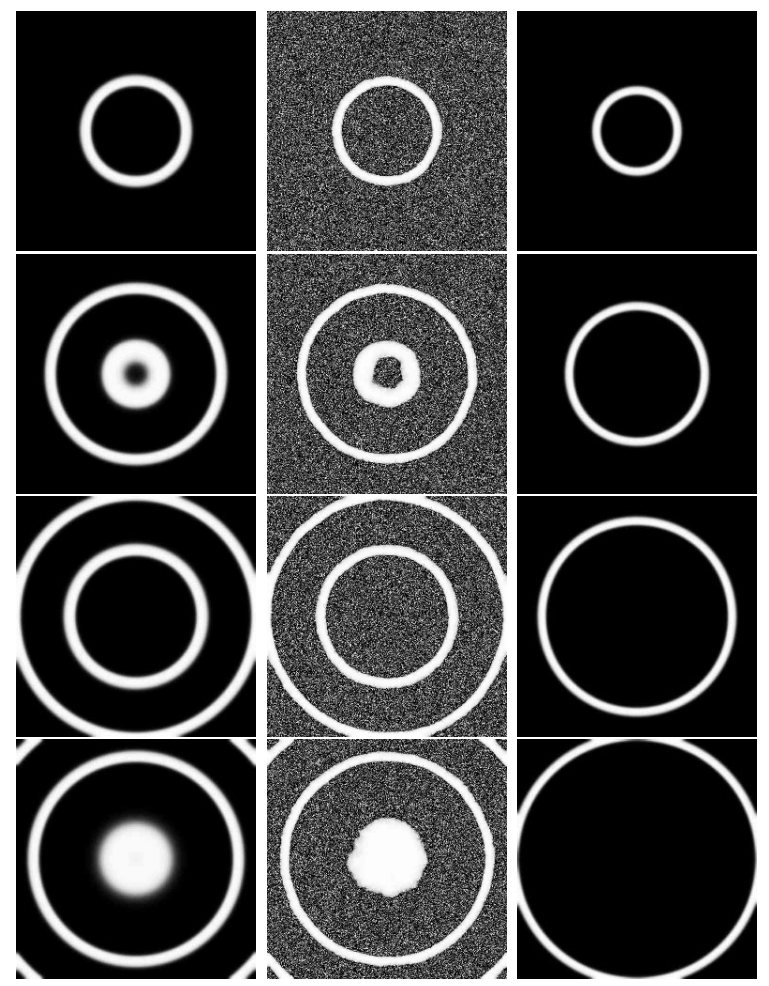

Fig. 3. Two-dimensional excitable system with a circular-like initial condition. The first column corresponds to the effective model with $C(0)=0.001$. The second one corresponds to the same system with white noise $\left(\sigma^{2}=0.00001\right)$. The last column represents the evolution of the deterministic model in the excitable regime. The snapshots are taken at 0.03, 0.09, 0.15, and 0.18 t.u. respectively. $(256 \times 256$ pixels, $a=0.7, b=0.02, \epsilon=0.04, \Delta x=0.1$ s.u., $\Delta t=0.001$ t.u. $)$. 


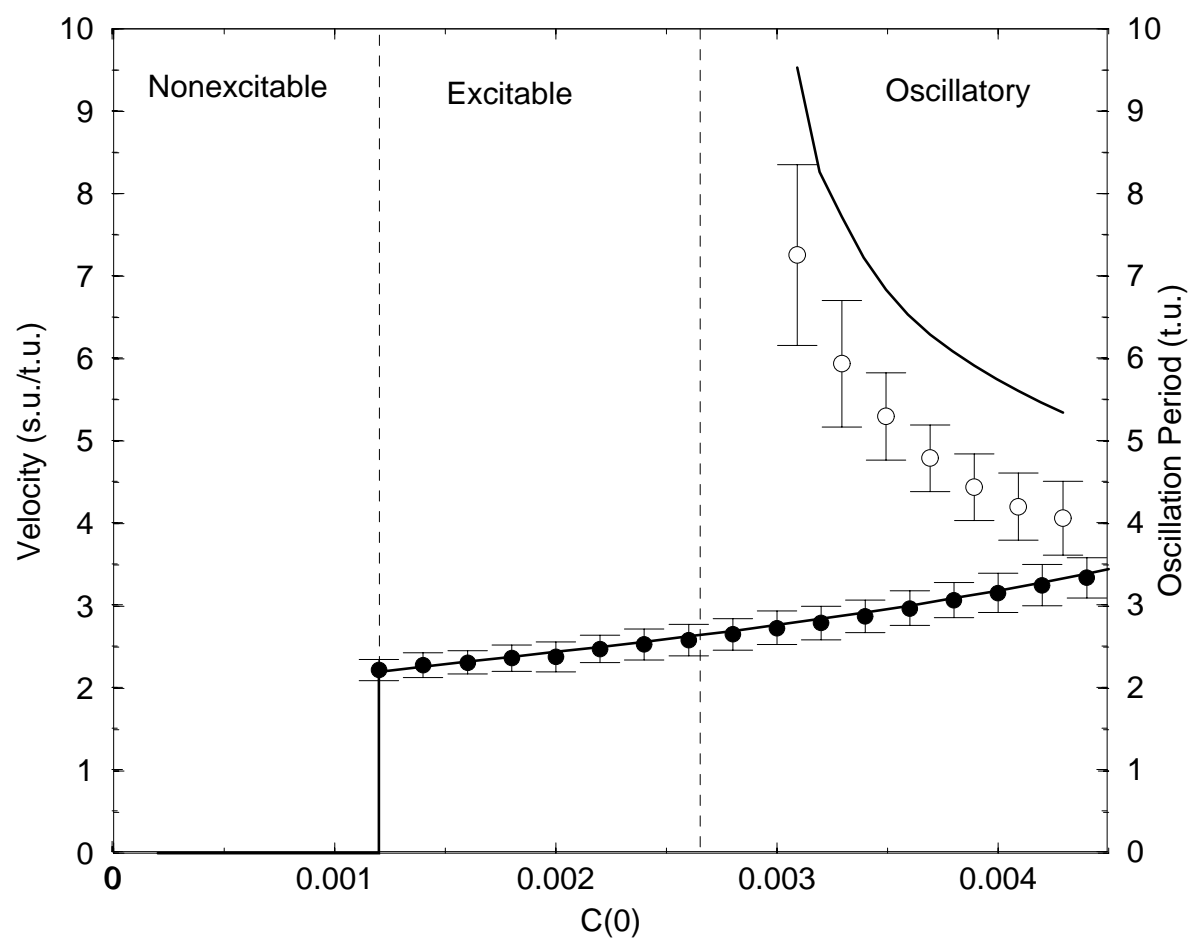

Fig. 4. Mean velocity $(\bullet)$ and mean oscillatory period (०) of noisy wave trains on a onedimensional nonexcitable media versus the noise intensity. Points calculated with an average of 25 realizations. Velocities and periods correspond to the effective model with the renormalized values of the parameter, Eqs. (12)-(16) (-). Parameters of the model are: $a=0.7, b=0.02$, $\epsilon=0.04, \Delta x=0.1$ s.u., $\Delta t=0.001$ t.u. Vertical lines show the transitions among different kinds of excitability regimes.

In order to characterize in a more quantitative form the transitions we have identified before, we have to choose a kind of order parameter whose drastic change would indicate the transition points. The transition from nonexcitable to excitable conditions can be defined quite easily by looking at the velocity of the wave: zero in the nonexcitable regime and nonzero in the excitable one. This criteria is useless to find the transition to the oscillatory regime. For this case we choose the period of the oscillations, or for practical purpose, the interval of time between two consecutive waves in a wave train. This period is not defined in nonexcitable and excitable regimes but it is finite in the oscillatory regime, diverging at the transition point. This analysis is shown in Fig. 4, by using numerical simulation data in onedimensional systems from the stochastic and effective models. These two transitions appear clearly and the effective model gives very reliable results. Main differences appear in the oscillatory regime due to the very large noise intensities used.

In all the cases if noise is switched off then the properties of the pattern recover the regime of the noise-free system. 


\section{Conclusions and Perspectives}

We have shown analytically and numerically that noise modeled in terms of Stochastic Partial Differential Equation can produce ordering effects. We have focussed our study on excitable model systems. Theoretical predictions have been checked satisfactorily by numerical simulations. Our results are not restricted to nonexcitableexcitable-oscillatory transitions. Recently we have proved that external fluctuations can also control a transition between bistable and excitable regimes. ${ }^{15}$

It is worth to comment here that in our analytical study we have assumed that the external noise is white in time and in space. This assumption has no problems in the lattice, but in the continuum description a colored noise in space has to be considered instead.

Certainly white noise assumption is a drastic simplification of the spatiotemporal structured nature of fluctuations in real experiments. Nevertheless experimental evidence of these noisy effects have been also tested in chemical experiments with the Belousov-Zhabotinsky reaction and, of course, with truly realistic noises. ${ }^{12}$ The theoretical analysis of this problem with real fluctuations or spatio-temporal structured noise has been done in Ref. 16 .

Thus we can conclude that the ordering effects of the external fluctuations are not a academic result but a real fact in nature.

\section{Acknowledgment}

We acknowledge financial support from the Dirección General de Enseñanza Superior (Spain) under projects BFM2000-0624 and BXX2000-0638.

\section{References}

1. J. García-Ojalvo and J. M. Sancho, Noise in Spatially Extended Systems (SpringerVerlag, New York, 1999).

2. T. Shinbrot and F. J. Muzzio, Nature 410, 251 (2001).

3. G. Giacomelli, M. Giudici, S. Balle, and J. R. Tredicce, Phys. Rev. Lett. 84, 3298 (2000).

4. J. M. Buldú, J. Garcí-Ojalvo, C. R. Mirasso, M. C. Torrent, and J. M. Sancho, Phys. Rev. E 64, 051109 (2001).

5. A. N. Zaikin and A. M. Zhabotinskii, Nature 225, 535 (1970).

6. R. Kapral and K. Showalter, Chemical Waves and Patterns (Kluwer, Dordrecht, 1995).

7. A. Goldbeter, Biochemical Oscillations and Cellular Rhythms (Cambridge Univ. Press, Cambridge, UK, 1996).

8. A. S. Mikhailov, Phys. Rep. 184, 307 (1989).

9. S. Kádár, J. Wang, and K. Showalter, Nature 391, 770 (1998).

10. I. Sendiña-Nadal, A. P. Muñuzuri, D. Vives, V. Pérez-Muñuzuri, J. Casademunt, L. Ramírez-Piscina, J. M. Sancho, and F. Sagués, Phys. Rev. Lett. 80, 5437 (1998).

11. I. Sendiña-Nadal, S. Alonso, V. Pérez-Muñuzuri, M. Gómez-Gesteira, V. Pérez-Villar, L. Ramírez-Piscina, J. Casademunt, J. M. Sancho, and F. Sagués, Phys. Rev. Lett. 84, 2734 (2000).

12. S. Alonso, I. Sendiña-Nadal, V. Pérez-Muñuzuri, J. M. Sancho, and F. Sagués, Phys. Rev. Lett. 87, 078302 (2001). 
13. M. A. Santos and J. M. Sancho, Phys. Rev. E 64, 016129 (2001).

14. D. Barkley, M. Kenss, and L. S. Tuckerman, Phys. Rev. A 42, 2489 (1990).

15. J. García-Ojalvo, F. Sagués, J. M. Sancho, and L. Schimansky-Geier, Phys. Rev. E. 65, 011105 (2002).

16. S. Alonso, F. Sagués, and J. M. Sancho, Phys. Rev. E 65, 066107 (2002). 